\title{
THE ELASTO-VISCOPLASTIC-TIME-DEPENDENT NATURE OF WAXY CRUDE OILS
}

\author{
B. A. Tarcha ${ }^{a}$, \\ B. P. P. Forte ${ }^{a}$, \\ E. J. Soares ${ }^{\text {a }}$, \\ and R. L. Thompson ${ }^{\mathrm{b}}$ \\ ${ }^{a}$ Universidade Federal do Espírito Santo \\ LabReo, Departamento de Engenharia Mecânica \\ Avenida Fernando Ferrari, 514 \\ CEP 29075-910, Vitória, Espírito Santo, Brasil \\ edson@ct.ufes.br \\ ${ }^{b}$ Universidade Federal Fluminense \\ LMTA, Departmento de Engenharia Mecânica \\ Rua Passo da Pátria, 156 \\ CEP 24210-240, Niterói, Rio de Janeiro, Brasil \\ Received: October 29, 2014 \\ Revised: November 30, 2014 \\ Accepted: December 30, 2014
}

\section{ABSTRACT}

Production in reservoirs located in deep and ultra-deep water that contain waxy crude oils faces a huge obstacle imposed by the low temperatures of the environment. When the waxy crude oil is subjected to a temperature below the Gelation Temperature, as in the case investigated in the present work, it exhibits a variety of non-Newtonian features: elasticity, plasticity, viscous effects, and time-dependency, which renders to this material a highly complex behavior. A crucial feature that is frequently ignored when the determination of the yield stress is being carried out is the timedependency nature of these materials. We demonstrate that this character has a significant impact on the measurement of the yield stress and, therefore, values obtained from a protocol that neglects time-dependency can be substantially different from a more careful procedure.

Keywords: waxy crude oils; re-start problem; yield stress measurement; time-dependent material

\section{NOMENCLATURE}

\section{Greek symbols \\ $\gamma_{\mathrm{c}} \quad$ critical strain \\ $\tau \quad$ shear stress}

\section{INTRODUCTION}

Oil basins at deep and ultra-deep water are becoming an important source of oil production nowadays. The temperatures in this kind of environment can achieve $4^{\circ} \mathrm{C}$. Hence, when production is interrupted for any reason, the oil cools down to temperatures below the Gelation Temperature (GT) and its paraffinic waxy nature induces a formation of crystals that form an interlocked network. In this gelled state, waxy crude oils exhibit a variety of non-Newtonian features: elasticity, viscoplasticity, time-dependency. Besides that, the rheological properties that describes this complexity nature are intrinsically dependent on the shear and temperature histories, adding another difficulty to the characterization of this kind of material.

The set of conditions needed in order to restore the flow after the oil has achieved the gelled state is usually called the re-start problem. Due to the economic impact of this issue to the petroleum industry, an effort on determining the yield stress of this state and, by consequence, defining its viscoplastic nature is considered of central importance. The understanding of the yielding behavior of waxy crude oils at temperatures below the GT is considered crucial for specifying the hydraulic plant of the oil production in deep and ultra-deep water.

Some of the difficulties associated to the determination of the rheological properties of waxy crude oils at low temperatures have been largely reported in the literature (Wardhaugh and Boger, 1991; Chang et al., 1998; Visitin et al., 2005; Dimitriou et al., 2011, Soares et al., 2013). One important aspect introduced is an extension of a Bingham-philosophical backbone where the goal is to determine the yield-stress of the material to a wider viewpoint where 1) different stages of yielding are detected, corresponding to: an elastic yield stress, a static yield stress, and a dynamic yield stress (Wardhaugh and Boger, 1991); 2) there is a high but finite viscosity associated to stresses below the yield stress, rendering a character of apparent-yield-stress, to the critical stress value where a major collapse of microstructure takes place (Barnes, 1999; Dimitriou et al., 2011). These two generalizations are engineering viewpoints to the problem of yield stress existance (Barnes andWalters, 1985; Astarita, 1990; Evans, 1992; Barnes, 1999; Møller et al., 2006; de Souza Mendes and Thompson, 2013).

\section{EXPERIMENTS}


Rheometric measurements were carried out with a commercial stress controlled rheometer, model HAAKE MARS II, manufactured by Thermo Scientific, Germany, by using a standard cone-plate geometry, in which a sample of $1 \mathrm{ml}$ is required for each test. The samples of waxy crude oil were supplied by the Brazilian Oil Company (Petrobras). The oil, light and free of salt and water contains, at least, $3 \%$ of asphaltenes and resins.

All samples need to be submitted to the same step-by-step procedure before any experiment. First of all, each sample was heated from $25^{\circ} \mathrm{C}$ up to $50^{\circ} \mathrm{C}$. This level of temperature was kept for 30 minutes. After that, using a precise thermal control equipment (HAAKE Phoenix II), the oil temperature was decreased at a constant rate of $0.6^{\circ} \mathrm{C} / \mathrm{min}$ until the test temperature of $4^{\circ} \mathrm{C}$ was reached. The sample rested for 3 hours at this temperature before the beginning of the test. In the beginning the paraffin crystals formation is quite intense. In the end of the 3 hours, the paraffin formation is substantially reduced and the oil is fully waxed. The total period of time before the test start up took 4 hours and a half.

A great number of tests were carried out until the point where the results obtained were more consistent and reproducible. In order to explore the characterization of this material in a broad sense, the tests were conducted by the two possible procedures: the controlled stress and the controlled strain modes. It is worthy of noting that the rheometer used in this investigation is, in fact, a controlled stress equipment. Thus, in the strain controlled mode, strain is truly specified by stress feedback. The main challenge of this kind of measurement is the time-dependency nature of the waxy crude oil. Therefore, extra care is needed to set up the necessary time interval for each point to achieve steady state conditions either in stress or in strain controlled mode. Typically, the data can be acquired continuously or step-by-step. The obtained results clearly indicate that the use of the step-by-step approach provides a more accurate test control, i.e. using the step-by-step manner of acquiring the data is easier to detect if the steady state regime is reached or not.

\section{RESULTS AND DISCUSSION}

This brief section reports different pictures of the complexity of waxy crude oils.

In Fig. 1 we notice a yield-stress behavior. For constant input values of stress (blue triangles) there is a jump on the shear rate values, showing a strong decrease in microstructure. The difference between this curve with the one obtained for constant input values of strain rate (red hollow triangles) is still under investigation, but it is probably related to shear banding. We notice that there is a reasonable agreement between these curves in the two extreme regimes of low and high shear rates.

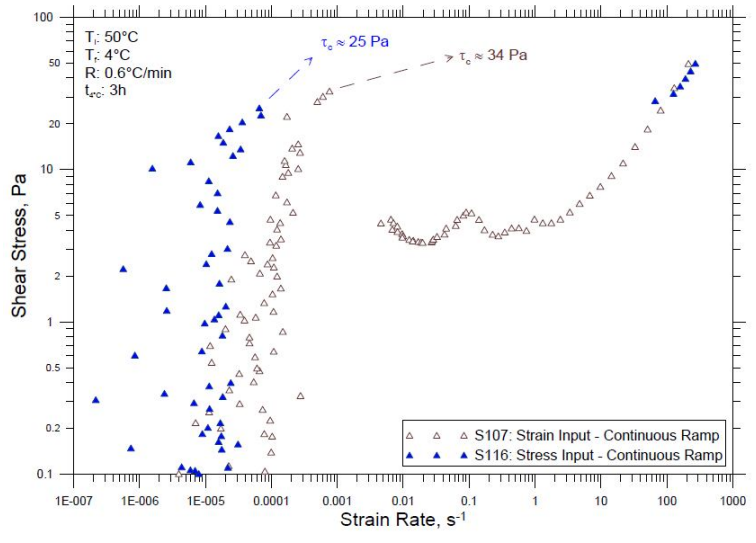

Figure 1. Shear stress as a function of shear rate for input values of stress and input values of shear rate.

Figure 2 shows the elastic behavior this waxy crude oil presents for low values of stress. Before a critical stress where an abrupt change occurs, we find a linear behavior between stress and strain, typical of constant elastic modulus elastic material. After a certain value of the stress there is a change in this linear behavior followed by an abrupt increase of the strain, which can be related to yielding. This brief section reports different pictures of the complexity of waxy crude oils.

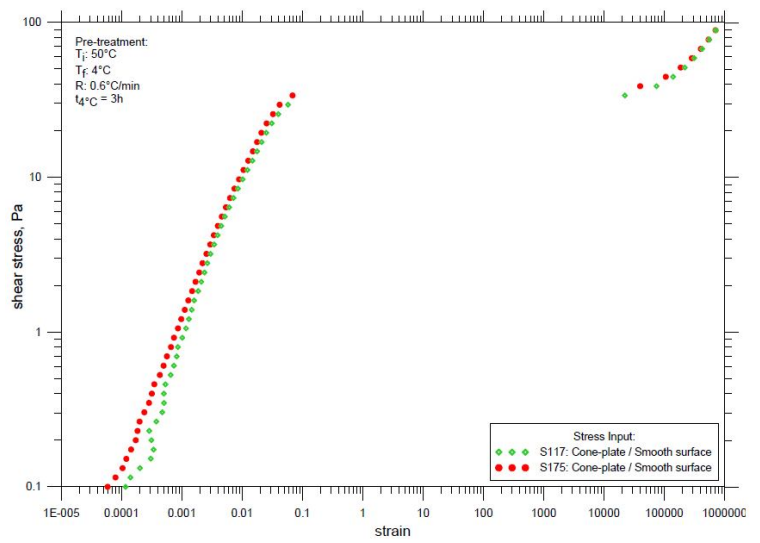

Figure 2. Shear stress as a function of shear strain for input values of stress.

Another very interesting behavior is shown in Fig. 3. There we can see the time-dependent nature of the tested waxy crude oil. A bifurcation occurs marking two different sets of behaviors: for high shear stress values, there is a typical viscous behavior, where a constant strain rate is obtained for a constant imposed stress. For low values of stress, a steady state is never reached, showing that for these levels of stress the material has not broken in the elapsed time evaluated. An intermediate case, $\tau=25$ $\mathrm{Pa}$, shows a different behavior. In this case, the material starts in the low shear stress group and suddenly detaches from this group evolving in time towards the viscous group. This behavior is typical 
from time-dependent materials. We notice that the amount of time taken to migrate from one group to the other is significant, $100 \mathrm{~s}$.

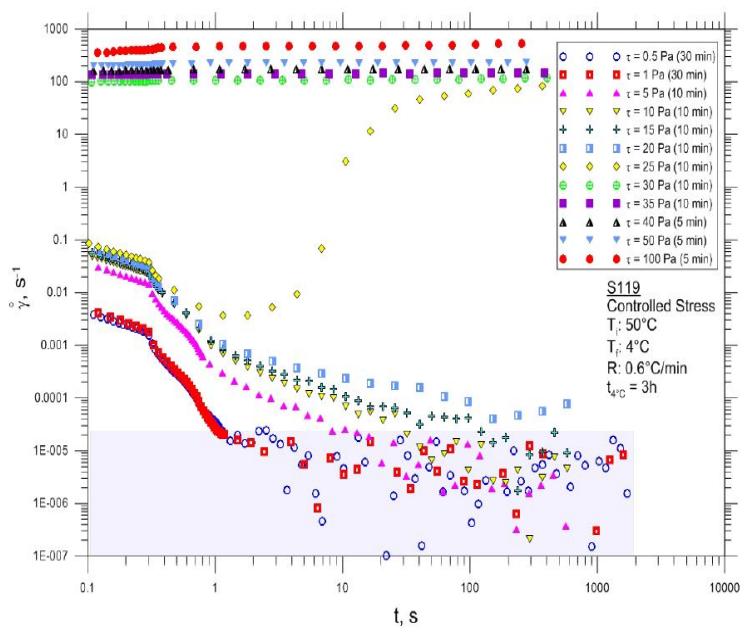

Figure 3. Shear rate as a function of time for input values of stress.

It remains to show if this time dependency is reversible (typical of thixotropic materials) or irreversible. Although there is an intrinsic difficulty on doing such analysis, namely to prove an irreversible behavior one has to wait an infinite amount of time, we can show that even waiting half an hour for each point, the totally destructed state of constant low viscosity continues when we decrease the shear stress after we have reached a maximum value. There is a quite linear behavior in this case.

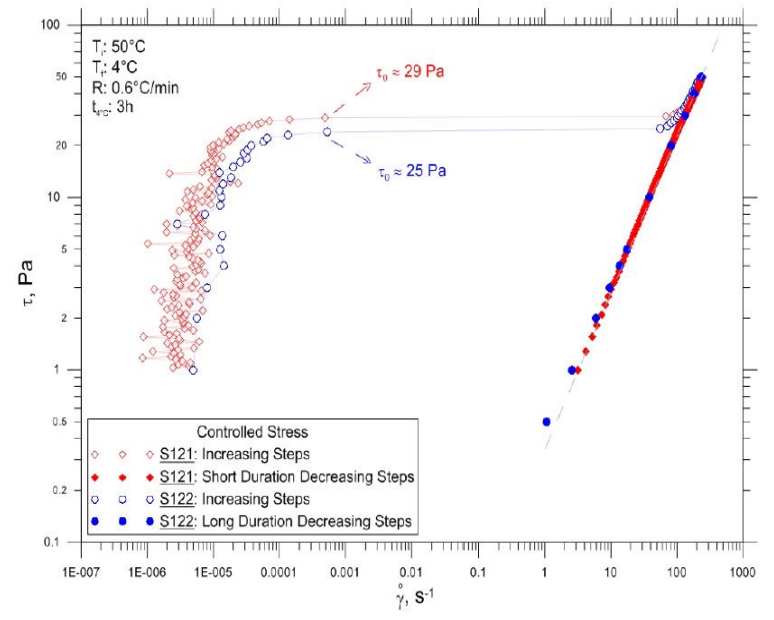

Figure 4. Irreversibility in the reverse experiment.

Two important figures shown below attest the main finding of the present work. It is the critical strain value. For different rupture stresses, we found a remarkable constant critical strain value associated to a major rupture.

Figure 5 shows the strain evolution in time for different values of imposed stress. We notice that different levels of stress induce solid-like to liquid like transition. This happens because each sample begins with its maximum structure level and, therefore, any stress value above the minimum level to induce breakage will trigger this transition. Until a certain point in time, the different samples remain in a solid-like trajectory without breaking. If we decided to wait until $\mathrm{t}=100 \mathrm{~s}$ one would come to the conclusion that $\tau=25 \mathrm{~Pa}$ was the best candidate to be considered the static yield stress of the material. However, time proved that, maintaining the stress level at $\tau=21 \mathrm{~Pa}$ for a longer period induces a major break up at time $\mathrm{t} \approx 300 \mathrm{~s}$. As explained by de Souza Mendes and Thompson (2012) when the stress level is above the yield stress, the intensity of this quantity (and not the intensity of shear rate) is crucial to determine the time scale associated to the breaking down process. Hence, not only the destruction process happens before, but also more rapidly. We notice that the very similar, but lower, stress level of $\tau=20 \mathrm{~Pa}$ gave no sign of inducing the breakage of the material until $t=104 \mathrm{~s}$. This waiting time is related to the limitations discussed in the preliminary results.

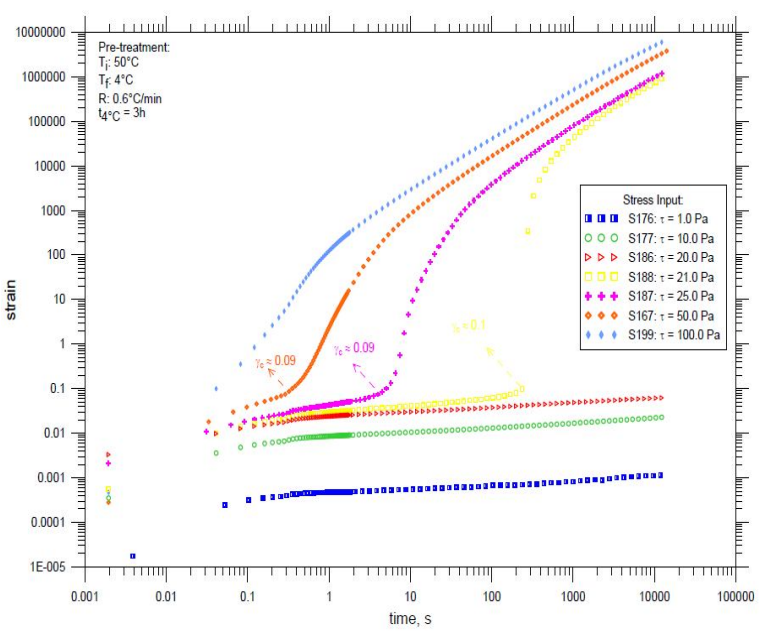

Figure 5. Strain as a function of time for different values of imposed stress applied to different samples.

In Fig. 6, we can see the strain as a function of time for imposed shear rate values. There, we can find a remarkably constant $\gamma_{\mathrm{c}} \approx 0.08$ value, maintained with a quite low deviation for all the samples tested. This result also corroborates the one obtained imposing different stress constant values, displayed in Fig. 5. It is interesting to notice the coincidence of the value obtained for the critical strain for these different tests. Interestingly, the material experiences a major collapse at $\gamma_{\mathrm{c}} \approx 0.1$ irrespectively of the stress level or the shear rate level. In other words, as long as the yield stress is overcome, the material destruction begins timid until it reaches a critical strain, where a severe rupture takes place. 


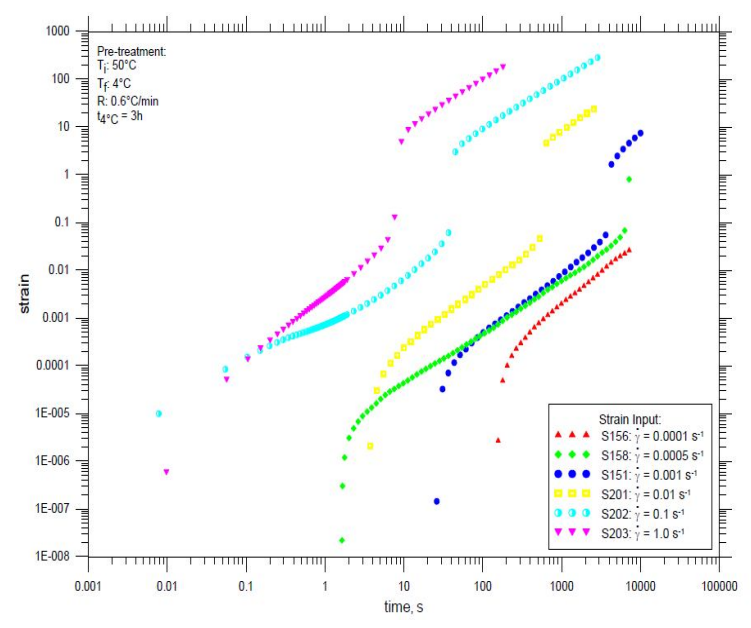

Figure 6. Strain as a function of time for different values of imposed strain rates applied to different samples.

Figure 7 shows the shear stress as a function of time for different values of imposed strain rates. This figure clearly shows the different levels of critical shear stress where a major rupture takes place after a linear elastic behavior. For the lowest value of constant shear rate imposed the yield stress was not achieved and no rupture is identified.

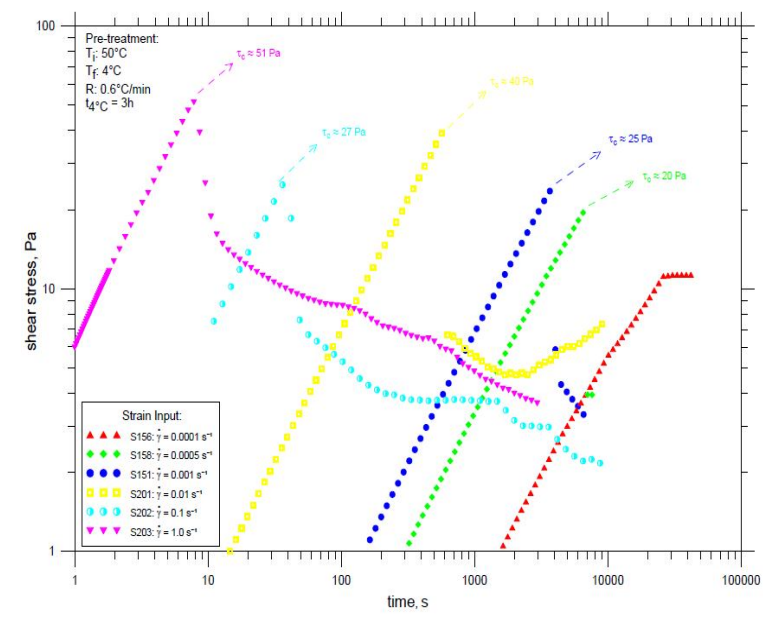

Figure 7. Stress as a function of time for different values of imposed strain rates applied to different samples.

\section{CONCLUSIONS}

In the present work we explore the rheology of a waxy crude oil which exhibits highly complex features in low temperatures. Elasticity, plasticity, time-dependency, are some of the non-newtonian characters this material presents.

We conducted constant stress and constant shear rate experiments for a single sample and for different samples in order to construct the history of the material. The time-dependency nature of this material is clearly demonstrated, a feature that only recently has received attention in the literature.

The main finding of the present work is to show that the critical strain where we can detect a major rupture is a more characteristic fingerprint of the material than the yield stress. This result will be better explored in the future.

\section{REFERENCES}

Astarita, G., 1990, The Engineering Reality of the Yield Stress, Journal of Rheology, Vol. 34, No. 2, pp. 275-277.

Barnes, H. A., 1999, The Yield Stress-a Review, Journal of Non-Newtonian Fluid Mechanics, Vol. 81, pp. 133-178.

Barnes, H. A., and Walters, K., 1985, The Yield Stress Myth?, Rheologica Acta, Vol. 24, pp. 323-326.

Chang, C., Nguyen, Q. D., and Bogger, D. V., 1998, The Yielding of Waxy Crude Oils, Industrial \& Engineering Chemistry Research, Vol. 37, pp. 15511559.

de Souza Mendes, P. R., and Thompson, R. L., 2012, A Critical Overview of Elasto-Viscoplastic Thixotropic Modeling, Journal of Non-Newtonian Fluid Mechanics, Vol. 187-188, pp. 8-15.

de Souza Mendes, P., and Thompson, R., 2013, A unified Approach to Model Elasto-Viscoplastic Thixotropic Yield-Stress Materials and Apparent Yield-Stress Fluids, Rheologica Acta, Vol. 58, pp. 1630-1640.

Dimitriou, C. J., McKinley, G. H., and Venkatesan, R., 2011, Rheo-Piv Analysis of the Yielding and Flow of Model Waxy Crude Oils, Energy \& Fuels, Vol. 25, pp. 3040-3052.

Evans, I. D., 1992, On the Nature of the Yield Stress, Journal of Rheology, Vol. 36, No. 7, pp. 13131316.

Møller, P. C. F., Mewis, J., and Bonn, D., 2006, Yield Stress and Thixotropy: on the Difficulty of Measuring Yield Stresses in Practice, Soft Matter, Vol. 2, pp. 274-283.

Soares, E. J., Thompson, R. L., and Machado, A., 2013, Measuring the Yielding of Waxy Crude Oils Considering its Time-Dependency and Apparent-Yield-Stress Nature, Applied Rheology, Vol. 23, pp. 62798-1, 62798-11

Visitin, R. F. G., Lapasin, R., Vignati, E., D'Antona, P., and Lockhart, T. P., 2005, Rheological Behavior and Structural Interpretation of Waxy Crude Oil Gels, Langmuir, Vol. 21, pp. 6240-6249.

Wardhaugh, L. T., and Boger, D. V., 1991, The Measurement and Description of the Yielding Behavior of Waxy Crude Oil, Journal of Rheology, Vol. 35, pp. 8123-8133. 